\title{
Mouth rinsing and ingesting pickle juice are no more effective than water for inhibiting electrically induced muscle cramps
}

\author{
Julia Georgieva 1, Carly J. Brade ${ }^{1}$, Kagan J. Ducker ${ }^{1}$, Paul Davey ${ }^{1}$, Angela Jacques ${ }^{1}$, Masato Ohno ${ }^{2}$ and Andrew P. \\ Lavender $3, *$ \\ Curtin School of Allied Health, Curtin University, Perth, Western Australia, Australia \\ 2 Department of Liberal Arts, Yonago College, National Institute of Technology, Yonago, Tottori, Japan \\ 3 School of Science, Psychology and Sport, Federation University Australia, Ballarat, Victoria, Australia \\ * Correspondence: a.lavender@federation.edu.au; Tel.: +61 353276593
}

\begin{abstract}
Background: Stimulating oropharyngeal transient receptor potential (TRP) channels inhibits muscle cramping by triggering a supraspinal reflex to reduce $\alpha$-motor neuron hyperexcitability. This study investigated whether longer stimulation of TRP channels via mouth rinsing with PJ is more effective than drinking PJ at inhibiting an electrically induced muscle cramp (EIMC); (2) Methods: Tibial nerve in 11 cramp-prone adults were percutaneously stimulated to elicit an EIMC of flexor hallucis brevis in three trials 1-week apart. At cramp onset participants received mouth rinsing and expelling PJ (25 mL), ingesting PJ ( $1 \mathrm{~mL} \cdot \mathrm{kg}^{-1}$ body-mass [BM]), or ingesting water $(1$ $\left.\mathrm{mL} \cdot \mathrm{kg}^{-1} \mathrm{BM}\right)$. Cramp onset and offset by electromyography and severity of discomfort was recorded using a visual analogue scale (VAS); (3) Results: Median time to cramp cessation as a percentage of water was $82.8 \% \pm 14.634$ and $68.6 \% \pm 47.782$ for PJ ingestion and mouth rinse respectively. These results had large variability and no statistically significant difference was observed. There were also no differences in perceived cramp discomfort between conditions, despite hazard ratios for time to VAS $=0$ higher than water for PJ ingestion (22\%) and mouth rinse $(35 \%)(\mathrm{p}=0.66$ and 0.51 respectively); (4) Conclusions: Data suggest no difference in cramp duration and perceived discomfort between PJ and water.
\end{abstract}

Keywords: Cramping; electromyography; electrical stimulation; cramp induction; flexor hallucis brevis; pickle juice; oropharyngeal transient receptor

\section{Introduction}

Exercise associated muscle cramps (EAMC) are involuntary, spasmodic, often painful contractions of skeletal muscles, that can last several minutes $[1,2]$. They generally occur in multi-joint muscle groups, such as the hamstrings or gastrocnemius, during or following exercise, when the muscles are active and shortened [1,3] (e.g., hip extension with knee flexion may cause a hamstring cramp). These cramps are common amongst recreational, and competitive athletes of various sports and ability levels [3-5] They are associated with elevated concentrations of muscle damage biomarkers [6] and local muscle fatigue, thereby impairing function [4].

There has been conjecture regarding the cause of EAMC, with the 'electrolyte-depletion and dehydration theory', and the 'altered neuromuscular control theory' predominating [2,3,7-11]. The former theory is based on anecdotal reports dating back over a century $[10,12]$, which postulate that EAMC are due to fluid and electrolyte depletion through exercise induced sweating [3]. It is believed that sweat loss leads to a reduction in plasma volume with exercise [8], which causes contracture of the interstitial space [13,14]. Recent research suggests that the pathophysiological mechanism triggering EAMC is multifactorial, however, the primary cause is believed to be the result of altered neuromuscular control $[9,14,15]$. The 'altered neuromuscular control theory' postulates that neuromuscular fatigue and muscle overload cause an imbalance in inhibitory and excitatory impulses to the muscle, causing EAMC $[1,9,10]$. Given that EAMC generally occur at shortened muscle 
length, reduced tension in the tendon may decrease inhibitory feedback from the Golgi tendon organ afferents $[1,9]$ which, when coupled with the excitatory impulses from muscle spindles, cause an imbalance between inhibitory and excitatory drives to the " $\alpha$ " -motor neurons $[1,9,10,16]$. This leads to greater excitability at the spinal level, and enhanced " $\alpha$ "-motor neuron discharge to the muscle fibres $[1,16]$. Initially, this presents as a muscle fasciculation when measuring electromyographic (EMG) activity; however, a localised muscle cramp may develop if fatiguing exercise persists [7].

Many potential remedies (e.g., stretching, massage therapy, kinesio-taping) have been developed to treat EAMC with varying levels of efficacy [14]. Ingestion of pickle juice $(\mathrm{PJ})$, a solution containing acetic acid and high concentrations of salt has become popular $[10,14]$. Miller et al. [17] reported that ingesting $1 \mathrm{~mL} \cdot \mathrm{kg}-1$ body-mass (BM) of PJ during an electrically induced muscle cramp (EIMC) reduced cramp time by up to $45 \%$. Given that EIMCs are strongly correlated with EAMC [16], the authors concluded that the inhibitory effects of PJ on EAMC may be caused by acetic acid [17]. The sour taste of acetic acid is thought to stimulate the oropharyngeal receptors to trigger a supraspinal reflex that increases inhibitory neurotransmitter activity, potentially activating interneurons to postsynaptically inhibit the " $\alpha$ " -motor neuron pool of the cramping muscle [17]. This notion would support the 'altered neuromuscular control theory', however, evidence for this is lacking.

The oropharynx is host to a vast array of receptors, including taste receptors [18] through which the peripheral gustatory system can gather multisensory information, and communicate this via neural pathways to the brainstem [18]. Transient receptor potential (TRP) channels are nociceptor ion channels within the oropharynx [19]. Although little is known about these receptors $[20,21]$, they are found to be stimulated by weak acids such as acetic acid [22]. Based on this neural mechanism, studies have assessed mouth rinse for preferentially stimulating taste receptors to elicit a physiological response [23-26]. For example, mouth rinsing with a carbohydrate solution triggers a neural response resulting in an ergogenic effect on endurance exercise performance [23,25]. The mouth rinse modality has gained popularity as a means of intervention delivery [24], and may be an appropriate modality for PJ delivery during muscle cramping to cause the inhibition of EAMC via stimulation from acetic acid [17]. Therefore, this study investigated whether mouth rinsing PJ: 1) reduced the duration of an EIMC compared with water, 2) reduced the discomfort associated with an EIMC more than water, and 3) was superior to ingesting PJ at inhibiting an EIMC in active cramp-prone adults.

\section{Materials and Methods}

\subsection{Participants}

Nineteen currently active adults aged 18-35-y volunteered to participate in this study. All participants were completing three sessions of exercise per week throughout the study. Only volunteers who had experienced EAMC within the past six months were considered for the study. Volunteers were excluded from participating in the study if they reported: 1) any lower limb injury or surgery within the past six months; 2 ) any past adverse reactions to electrical stimulation; 3 ) an allergy to pickles; 4 ) having been diagnosed with any cardiovascular, neurological, or blood-borne pathologies, as electrical stimulation may trigger adverse physiological processes in these conditions [27]. Participants (n $=8$ ) were further excluded if they failed to experience an EIMC in their flexor hallucis brevis (FHB) when stimulated at $24 \mathrm{~Hz}$ during the familiarisation session.

Therefore, the final participant number was 11 (females $\mathrm{n}=3$; mean $\pm \mathrm{SD}$, age $=27.1 \pm 5.0$ $\mathrm{y}$, height $=176.8 \pm 9.2 \mathrm{~cm}, \mathrm{BM}=76.3 \pm 14.4 \mathrm{~kg}$ ). All procedures were approved by the Curtin University Human Research Ethics Committee (HRE2018-0651), and all participants provided written informed consent. 


\subsection{Study Design}

A randomised, repeated measures crossover design was used to ascertain the effect of three conditions: 1) mouth rinsing $25 \mathrm{~mL}$ of PJ (Pickle Juice Sport, The Pickle Juice Company, Mesquite, TX, USA) for $10 \mathrm{~s}$, then expelling [24]; 2) ingesting $1 \mathrm{~mL} \cdot \mathrm{kg}-1 \mathrm{BM}$ of PJ (Pickle Juice Sport, The Pickle Juice Company, Mesquite, TX, USA); 3) ingesting $1 \mathrm{~mL} \cdot \mathrm{kg}$ $1 \mathrm{BM}$ of room temperature water [17], on cramp duration, and severity of discomfort experienced from an EIMC in the FHB of active cramp-prone adults. Pickle juice mouth rinse and ingestion will be henceforth referred to as PJMR, and PJI respectively.

\subsection{Experimental procedures}

\subsubsection{Familiarisation session}

Participants attended a familiarisation session where the study procedures were explained, and to screen whether electrical stimulation of their FHB was tolerated, and productive of a cramp. The FHB was selected because low frequency percutaneous stimulation of this muscle induces cramps that have both intra- and inter-sessional reliability $[17,28]$, The non-dominant leg was screened for cramp induction during familiarisation, whilst the dominant leg was stimulated during testing [17]. This prevented any residual local physiological changes from the familiarisation session affecting the results during testing, which commonly occurred the following day [17]. The participants dominant leg was determined by asking the participant which is their preferred kicking leg.

During the familiarisation session, the participant sat upright on a plinth with their legs fully extended and ankles placed over the edge. On the non-dominant leg, two $\mathrm{Ag} / \mathrm{AgCl}$ EMG electrodes were positioned $2 \mathrm{~cm}$ apart on the FHB mid-belly [28], and a reference electrode was placed on the ipsilateral lateral malleolus using standard EMG preparatory procedures [29] (see Figure 1). This leg was then prepared for cramp induction using electrical stimulation with a constant current stimulator (model DS7A, Digitimer Ltd, Hertfordshire, UK). The stimulation conductor was positioned inferior to the medial malleolus (Figure 1), and the tibial nerve was stimulated sub-maximally 2 - 4 times, with a $1 \mathrm{~ms}$ electrical stimulus at $200 \mathrm{~mA}$. The site which stimulated the greatest hallux flexion was marked for replication during the cramp induction. The EMG electrodes were then connected to a computer via a Bortec AMT-8 EMG amplifier (Bortec Biomedical Ltd, Calgary, Canada) and an NI USB-6221 analogue to digital converter (National Instruments, Austin, TX, USA) which began recording time and EMG from the initiation of the electrical stimulus, using LabView software package (National Instruments, Texas, USA).
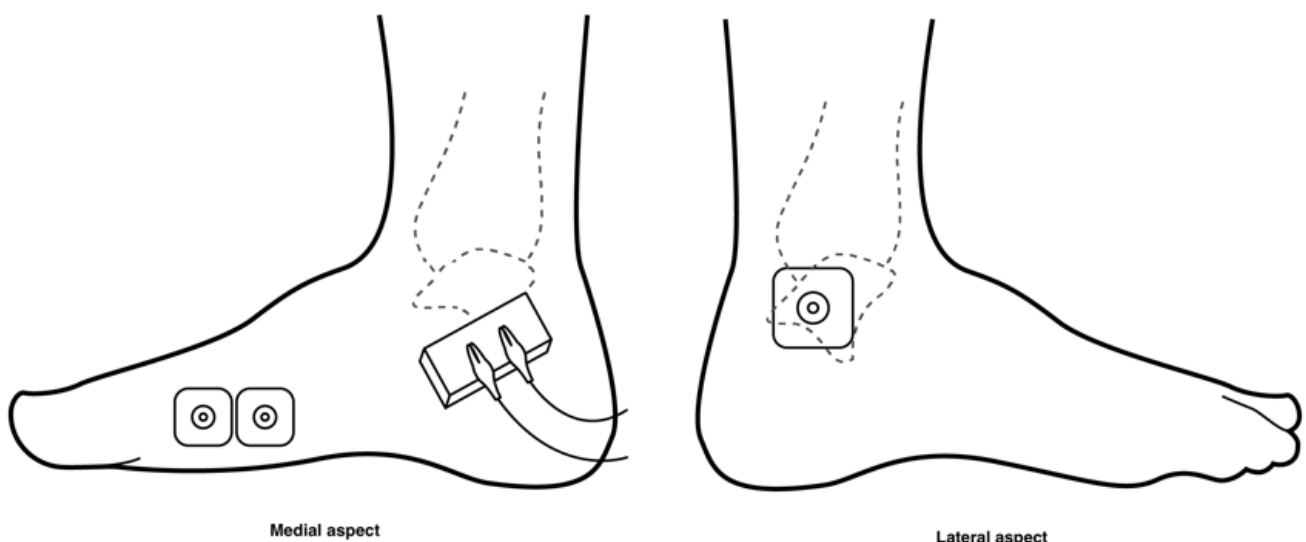

Figure 1. Diagram of electrode and stimulation conductor placement.

The participant was shown how, and practiced performing an isometric contraction of their non-dominant FHB. Once able, they were instructed to repeat and maintain the contraction until signalled to relax, and were told to verbalise when they felt the muscle was about to cramp. When the impending cramp was signalled, a train of electrical stimulation via the tibial nerve was delivered at an intensity of $200 \mathrm{~mA}$, for $2 \mathrm{~s}$. The volitional contraction was maintained for $10 \mathrm{~s}$ from stimulus initiation, then the participant was 
instructed to relax. The initial stimulus frequency was $4 \mathrm{~Hz}$, delivering a total of eight stimuli on the first trial [28]. If a cramp was not induced, the participant was rested for 1 min and the process was repeated with the train frequency increased by $2 \mathrm{~Hz}$ [28]. This process was repeated until either an EIMC of the FHB was produced, or the frequency reached $24 \mathrm{~Hz}$ [28]. The frequency at which a cramp was induced was recorded as the participant's cramp threshold frequency (CTF), which is the minimum frequency required to stimulate a cramp [30]. This CTF was used to induce all subsequent cramps.

The FHB was considered cramping if involuntarily contraction immediately succeeded cessation of volitional contraction, verified by the participant's self-report of a cramp, sustained hallux flexion, and an average EMG root mean square amplitude $>2$ SDs greater than the baseline reading [28]. Upon determining the CTF, the protocols for ingesting and mouth rinsing were practiced with water, thus ensuring protocol familiarity prior to experimental sessions.

\subsubsection{Experimental sessions}

Assuring inter-sessional standardisation, participants attended all experimental sessions having fasted (12 hours) and abstained from exercise ( 24 hours). Upon arrival, a refractometer (Sper Scientific, Ltd, Scottsdale, USA) was used to measure urine specific gravity (USG) ensuring each participant was in a dehydrated state reading $>1.050$ [31], as dehydration may increase the likelihood of cramp onset [32].

To minimise effects between trials, testing sessions were performed on the dominant leg at least one week apart [17]. The leg was prepared as per the familiarisation session, and electrode positions were marked with permanent marker [28] and photographed to enable replication in subsequent trials. Participants were instructed to re-mark the sites if fading was observed [28]. During the initial testing session, prior to the first volitional contraction, baseline FHB activation was measured for $15 \mathrm{~s}$ while the volunteer relaxed the muscle. Before cramp induction, the participant was instructed to refrain from stretching the upcoming cramp, allowing maximum duration. At their pre-established CTF, a cramp was induced using a $200 \mathrm{~mA}$ stimulation for $2 \mathrm{~s}$. Immediately following cramp detection and verification (10 $\mathrm{s}$ after electrical stimulus initiation), the participant was signalled to administer the intervention (PJMR, PJI, or water ingestion). Every $10 \mathrm{~s}$ during the cramp, the participant was instructed to rate the severity of discomfort experienced on a $100 \mathrm{~mm}$ visual analogue scale (VAS), where $0=$ no discomfort and $10=$ maximal discomfort. A cramp was considered alleviated when the EMG activity returned to $\leq 5 \%$ of the baseline contraction for $3 \mathrm{~s}$.

\subsection{Statistical Analysis}

A one-way ANOVA was used to compare USG mean scores. To determine the effect mouth rinsing and ingesting PJ had on cramp duration, EMG raw data were rectified and digitised, and then individual values for PJMR and PJI were normalised to the water condition before being analysed. The EMG data was notch filtered at $50 \mathrm{~Hz}$ using a 2nd order zero lag Butterworth filter. The data were then smoothed using a moving root mean square window of $1 \mathrm{~s}$. The minimum of these smoothed data was located, and the mean of the surrounding $0.5 \mathrm{~s}$ smoothed data was calculated. The time point at which the smoothed data dropped below $1.5 \mathrm{x}$ the minimum smoothed value for $0.1 \mathrm{~s}$ was then located to determine the offset time of the cramp.

Linear mixed models with random intercept participant effects were used to compare VAS between conditions over time and results were summarised using graphical representation of the marginal estimates. Time to cramp-end and time to zero discomfort $(\mathrm{VAS}=0$ ) between conditions were estimated using Kaplan-Meier survival probabilities. Results were summarised using Kaplan-Meier curves and medians and 95\% confidence intervals of times to events. Log Rank tests were used to compare survival probabilities between conditions. Effect of condition on time to zero discomfort was examined using Cox proportional hazards regression, and effects summarised using hazard ratios and 95\% confidence intervals. Participants were censored if VAS did not reach zero. Longitudinal and mixed model data were analysed using Stata version 15.1 (StataCorp, TX, USA), 
all other data were analysed with IBM SPSS version 24.0 (IBM Corporation, New York, NY, USA). Statistical significance was accepted at $\mathrm{p}<0.05$.

\section{Results}

\subsection{Urine specific gravity}

There were no significant differences in USG between PJMR (mean \pm SD; $1.068 \pm$ $0.005), \mathrm{PJI}(1.067 \pm 0.007)$, and water $(1.066 \pm 0.01)$ conditions $(\mathrm{F}=0.069, \mathrm{P}=0.93)$.

\subsection{Cramp duration}

Median time to cramp cessation for the control (water) condition was $50.68 \mathrm{~s}$. As a percentage of the control condition, the median cramp duration of the PJI and PJMR conditions were $82.8 \%(95 \%$ CIs $=54.13-111.50)$ and $68.6 \%(95 \%$ CIs $=0.00-162.21)$ respectively (Table 1) based on the Kaplan-Meier survival probabilities (Figure 2). However, this time difference was not significantly different between conditions ( $p=0.942, \log -\operatorname{Rank}$ test). Furthermore, given that the confidence intervals were set at 100, and the ranges for both PJMR and PJI include the null value of 100, there was no statistical difference between conditions.

Table 1. Medians for cramp survival time

\begin{tabular}{|c|c|c|c|c|c|c|}
\hline \multirow[b]{2}{*}{ Condition } & \multirow{2}{*}{$\begin{array}{l}\text { Median } \\
\text { Estimate }\end{array}$} & \multirow[t]{2}{*}{ Point } & \multirow{2}{*}{$\begin{array}{l}\text { Median } \\
\text { Error }\end{array}$} & \multirow[t]{2}{*}{ Std. } & \multicolumn{2}{|c|}{$\begin{array}{l}\text { Median } \\
\text { 95\% Confidence Interval }\end{array}$} \\
\hline & & & & & Lower Bound & Upper Bound \\
\hline PJI & 82.81 & & 14.63 & & 54.13 & 111.50 \\
\hline PJMR & 68.56 & & 47.78 & & 0.00 & 162.21 \\
\hline Water & 100.000 & & 0.00 & & - & - \\
\hline Overall & 100.000 & & 0.34 & & 99.34 & 100.67 \\
\hline
\end{tabular}

Point estimate - expressed as a percentage of the water control condition, with water set at 100\%; Median $95 \%$ confidence intervals set at 100, therefore ranges must not cross 100 to be considered statistically different; PJI - pickle juice ingested; PJMR - pickle juice mouth rinsed. 


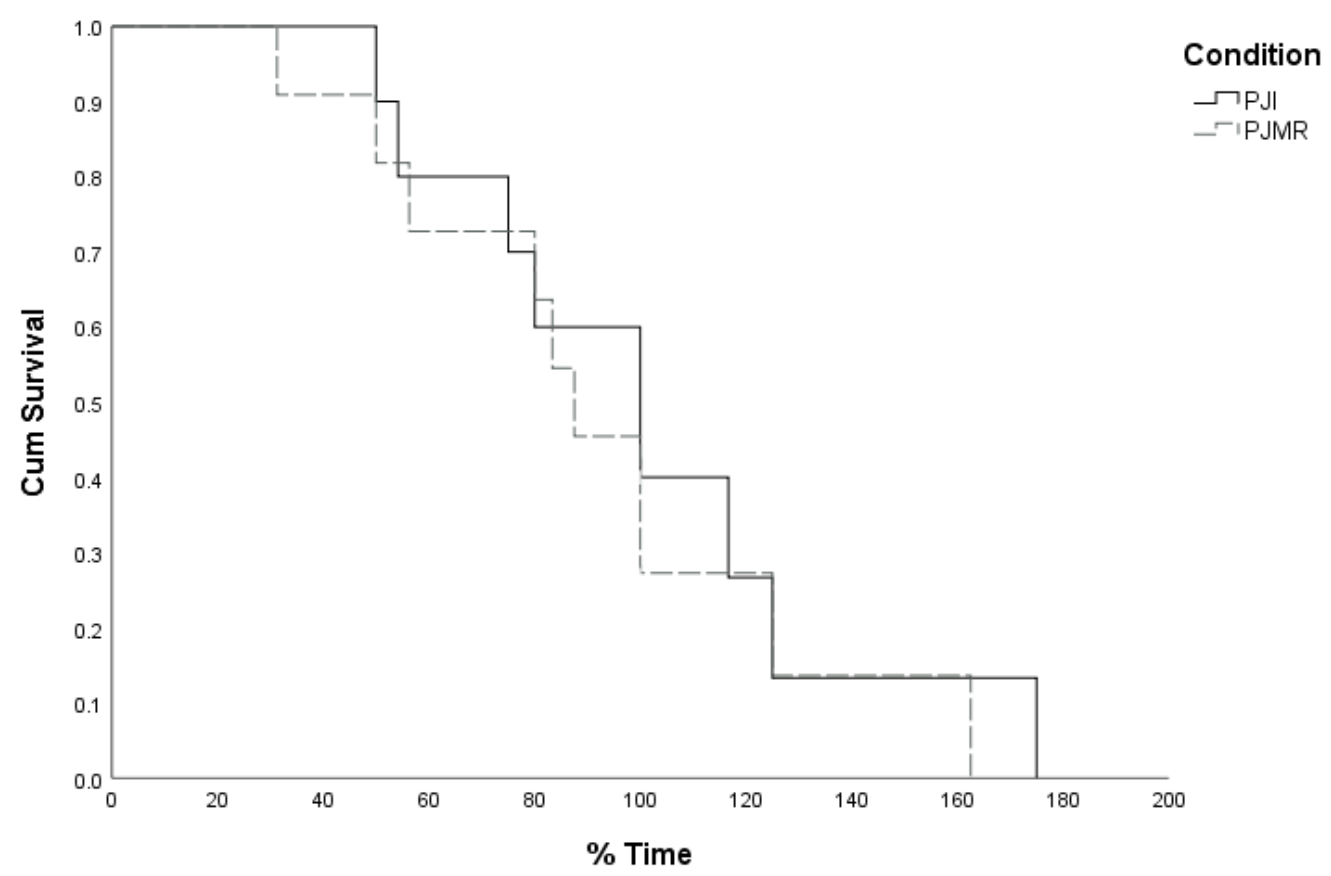

Figure 2. Kaplan-Meier (time to event) survival analysis showing time (expressed as a percentage of the water control condition, with water set at $100 \%$ ) to proportion cramping returns to 0 , representing cramp offset. PJI - pickle juice ingested; PJMR - pickle juice mouth rinsed.

\subsection{Severity and duration of perceived cramp discomfort (VAS)}

Linear mixed models with random subject intercepts were used to compare VAS between conditions over time (Figure 3). Kaplan-Meier survival probabilities showed no significant difference in time to VAS $=0$ between conditions ( $p=0.76$, Log Rank test) (Figure 4). Cox regression was used to compare PJI and PJMR to water for time to VAS $=0$. Although the hazard ratios for both PJI and PJMR were higher (22\% and 35\%) compared with water, these results were not statistically significant $(p=0.67$ and 0.51$)$. Note: One participant did not reach zero in all conditions and was therefore censored in the survival model. 


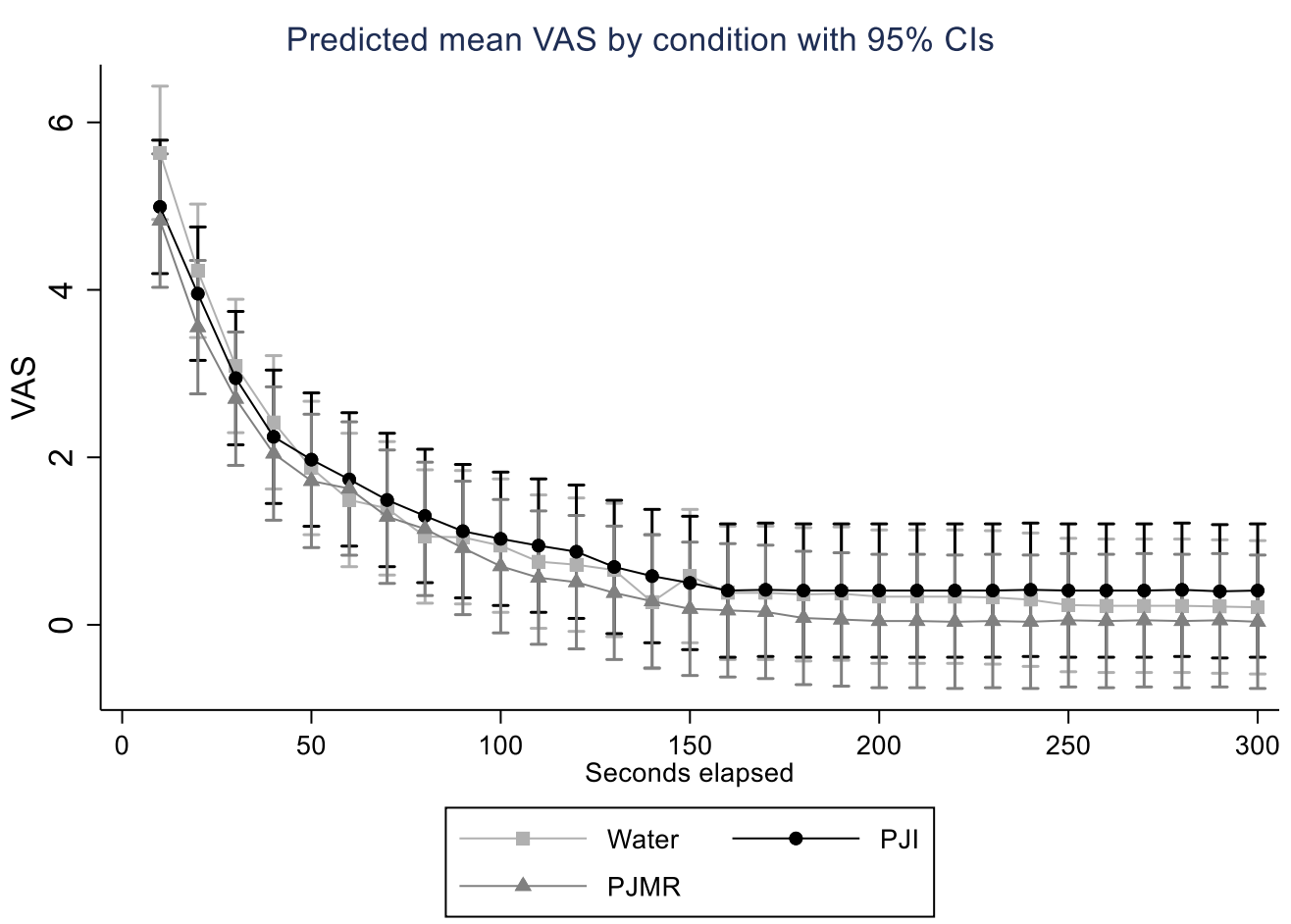

Figure 3. Linear mixed models with random subject intercepts comparing visual analogue scale (VAS) between conditions over time (seconds).

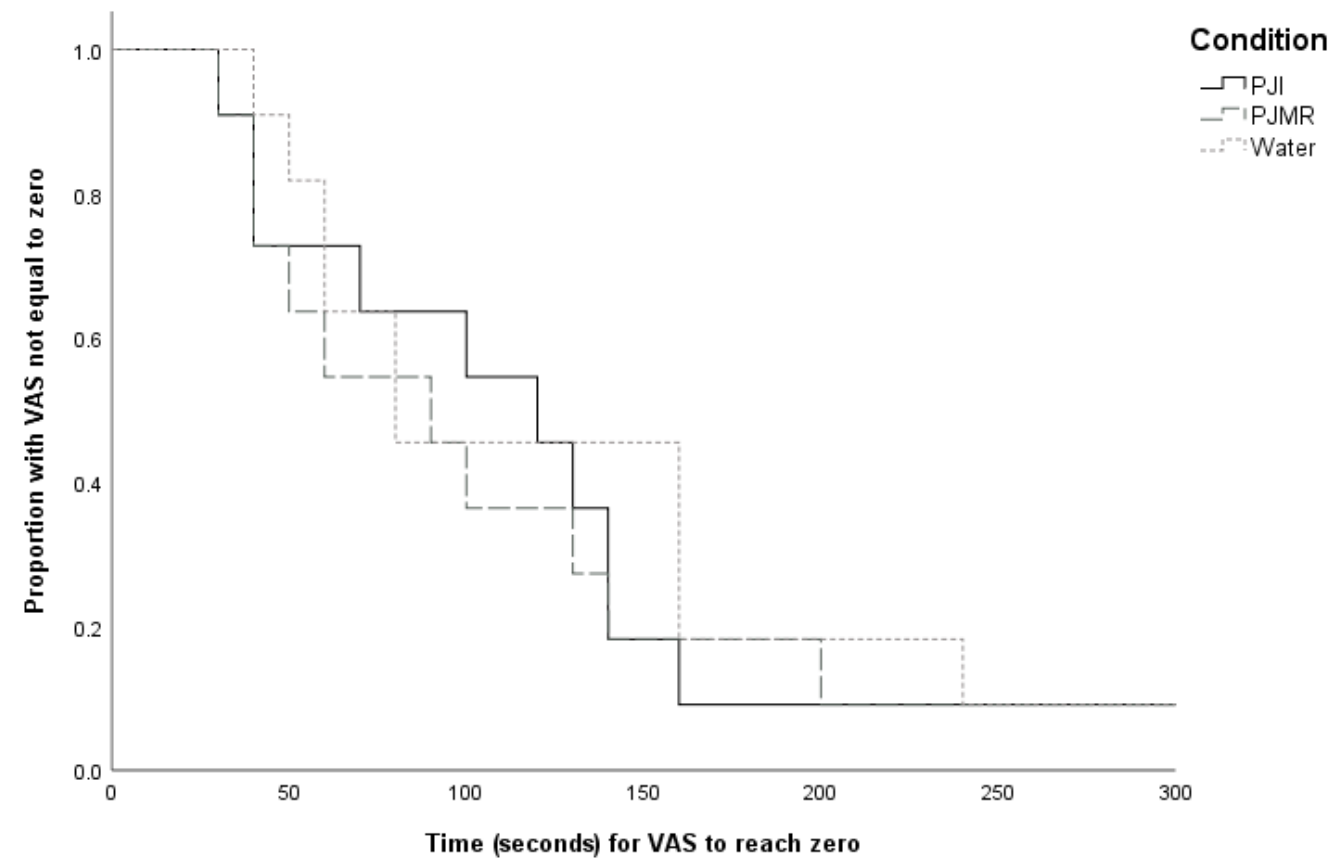

Figure 4. Kaplan-Meier (time to event) survival analysis for time (seconds) to visual analogue scale (VAS) to return to 0 , representing severity of discomfort.

\section{Discussion}

This study is the first to report whether mouth rinsing PJ has any inhibitory effect on EIMCs in active cramp-prone adults. It aimed to determine whether mouth rinsing PJ: 1) reduced the duration of an EIMC when compared with water, 2) reduced the discomfort 
associated with an EIMC more than water, and 3) was superior to ingesting PJ at inhibiting an EIMC.

There was no significant difference in cramp duration between conditions. Interestingly however, the point estimates for median cramp duration reduced by $17.2 \%$ and $31.4 \%$ in PJI and PJMR respectively compared with water (50.68 s). Similarly, Miller et al. [17] found that cramp duration was reduced by $45 \%$ compared with baseline when ingesting PJ (pre: $153.2 \pm 23.7$ s, post: $84.6 \pm 18.5$ s). A recent study investigating the acetic acid concentration in various substances found that several pickle brines (acetic acid weight percent $[\mathrm{AAwt} \%$ ] range $=0.406 \pm 0.007-0.796 \pm 0.007$ ] [33] similar to those used in the study by Miller et al. [17] - had a greater AAwt $\%$ than the proprietary PJ $(0.260 \pm$ $0.006 \%$ ) [33] used in the current study. Reduced acetic acid concentration in the tested conditions may suggest a larger sample was needed to account for concentration difference, potentially contributing to the lack of significance observed. Furthermore, unlike the current study, Miller et al. [17] only included cramps that lasted $\geq 90 \mathrm{~s}$, thus reducing the potential for variability. Although a similar sample and intervention was tested in the current study, the absence of a minimum cramp duration inclusion criterion resulted in wide-ranging variability between participants, making it difficult to assess any changes caused by PJ.

Miller et al.'s [17] study reported reliable electrical induction of long ( $\geq 90 \mathrm{~s})$ muscle cramps in the FHB of participants. However, despite replicating Miller et al.'s [17] cramp induction protocol during pilot testing, the authors were unable to induce muscle cramps reliably. Even upon modifying the protocol, the authors observed marked variability in cramp duration between participants, with few lasting $\geq 90 \mathrm{~s}$. Consistency in individuals' inter-sessional cramp durations was observed, resulting in the inclusion of all participants who experienced a FHB EIMC during the familiarisation session, regardless of cramp duration. Considering that few participants experienced long cramps, the current sample is a better representation of the general cramp-prone population.

Despite expanding the inclusion parameters, the authors were unable to induce cramps in eight cramp-prone participants during the familiarisation session, resulting in exclusion of these participants from the study thereby reducing the total number of datasets collected. Furthermore, the unsecured foot position of participants during testing may also contribute to the variability in the data. By not adequately restraining the foot there is the potential for adjacent muscles to cause reciprocal inhibition that affects FHB activation [34], possibly impacting the results. Improved methods for inducing and maintaining a cramp for long enough to assess the effects of interventions should be a priority for future research.

The current study showed no significant changes in severity or duration of perceived cramp discomfort between conditions during an EIMC. No other studies monitoring the effect an intervention had on perceived discomfort throughout the duration of a cramp have been identified. Other studies testing TRP-activator ingestion, assessed perceived discomfort severity pre- and post-cramp induction, but not during, with mixed findings $[30,35]$. One study found no changes to perceived discomfort between the TRP-activator intervention group and placebo control at various time points across the $24 \mathrm{~h}$ following the electrical induction of a calf cramp [30]. Whilst another found that during the initial 20 min following volitional calf cramp cessation, the perceived discomfort (on a 1 to 10 rating scale) was significantly lower for the TRP-activator group (3.4 \pm 4.6$)$ than the control $(4.0 \pm 2.2)$ [35]. Interestingly, Earp et al. [36] found that perceived discomfort severity of an EIMC was significantly $(\mathrm{p}=0.01)$ reduced in participants when comparing an electrolyte beverage $(2.00 \pm 0.54)$ to a placebo beverage $(2.88 \pm 0.84)$. However, unlike the current study, these study protocols had participants ingesting the conditions prior to cramp induction. Therefore, it is not yet known whether TRP-activators or other interventions reduce discomfort during or after muscle cramps when ingested immediately following cramp onset.

Muscle cramps are typically offset by passive stretching of the muscle secondary to pain and loss of function [14]. In this study participants were asked to relax and allow the 
cramp to subside unaided. The severity of discomfort reported by participants varied greatly and so it is not possible to suggest any relationship between severity or duration of discomfort and PJ.

Through assessing PJ mouth rinsing and ingestion, this study was able to measure the effects of exclusively stimulating oropharyngeal receptors with PJ. Although the literature suggests that $\mathrm{PJ}$ alleviates skeletal muscle cramping by stimulating oropharyngeal TRP-channels that trigger an inhibitory supraspinal reflex [17,19,30,37], the current study does not support these findings. The authors found no statistical difference in cramp duration or perceived discomfort between PJ and water in active cramp-prone adults.

Given the limitations of the study recommendations for future research can be suggested. The aetiology of EAMC is still unknown; however, it is likely multifactorial in nature with altered neuromuscular control [38] and hypohydration contributing to onset [32]. To better understand the mechanism causing immediate relief of EAMC, future studies should compare PJ with water by testing mouth rinsing and ingesting for both conditions. Furthermore, intervention and placebo groups should be considered to enable blinding. However, an appropriate placebo for PJ will be difficult to create and may present challenges with assessment.

\section{Conclusions}

The present study found no evidence that PJ ingested or mouth rinsed is more effective than water for alleviation of EIMC. However, given the large variability in cramp duration it is difficult to determine any meaningful effect of PJ on muscle cramps. Future studies should seek to determine the most consistent protocol for cramp induction, and continue research into alleviating EIMC quickly with an improved methodological approach to better understand whether mouth rinsing $\mathrm{PJ}$ is, in fact, an efficacious treatment for acute EAMC.

Author Contributions: Conceptualization, J.G., A.L., C.B., K.D. and M.O.; methodology, J.G., A.L., C.B., and K.D.; software, P.D.; validation, J.G., A.L., C.B., K.D., P.D., A.J. and M.O; formal analysis, A.J.; investigation, J.G. and A.L.; resources, J.G., A.L. and P.D.; data curation, P.D., J.G. and A.L.; writing-original draft preparation, J.G.; writing-review and editing, J.G., A.L., C.B., K.D., P.D., A.J. and M.O; visualization, J.G., A.J., A.L.; supervision, A.L., C.B. and K.D.; project administration, A.L. and J.G.; funding acquisition, A.L. All authors have read and agreed to the published version of the manuscript.

Funding: This research received no external funding.

Institutional Review Board Statement: The study was conducted according to the guidelines of the Declaration of Helsinki, and approved by the Ethics Committee of Curtin University (HRE20180651).

Informed Consent Statement: Informed consent was obtained from all subjects involved in the study.

Data Availability Statement: The data presented in this study are available on request from the corresponding author.

Acknowledgments: The authors would like to thank the participants in this study and Miss Eloise Arnold for her idea that initiated the project.

Conflicts of Interest: The authors declare no conflict of interest.

\section{References}

1. Schwellnus, M.P.; Derman, E.W.; Noakes, T.D. Aetiology of skeletal muscle 'cramps' during exercise: a novel hypothesis. J Sports Sci 1997, 15 (3), 277-85. doi:10.1080/026404197367281. 
2. Swash, M.; Czesnik, D.; de Carvalho, M. Muscular cramp: causes and management. Eur J Neurol 2019,26 (2), $214-21$. doi:10.1111/ene.13799.

3. Miller, K.C.; Stone, M.S.; Huxel, K.C.; Edwards, J.E. Exercise-associated muscle cramps: causes, treatment, and prevention. Sports Health 2010, 2 (4), 279-83. doi:10.1177/1941738109357299.

4. Minetto, M.A.; Holobar, A.; Botter, A.; Farina, D. Origin and development of muscle cramps. Exerc Sport Sci Rev 2013, 41 (1), 3-10. doi:10.1097/JES.0b013e3182724817.

5. Hoffman, M.; Stuempfle, K. Muscle cramping during a 161-km Ultramarathon: comparison of characteristics of those with and without cramping. Sports Medicine - Open 2015, 1 (1), 1-9. doi:10.1186/s40798-015-0019-7.

6. Martínez-Navarro, I.; Montoya-Vieco, A.; Collado, E.; Hernando, B.; Panizo, N.; Hernando, C. Muscle cramping in the marathon: dehydration and electrolyte depletion vs. muscle damage. J Strength Cond Res 2020, Publish Ahead of Print, 1-7. doi:10.1519/JSC.0000000000003713.

7. Schwellnus, M.P. Cause of exercise associated muscle cramps (EAMC) - altered neuromuscular control, dehydration or electrolyte depletion? Br J Sports Med 200943 (6), 401-8. doi:10.1136/bjsm.2008.050401.

8. Edouard, P. Exercise associated muscle cramps: discussion on causes, prevention and treatment. Sci Sport 2014, 29 (6) 299-305. doi:10.1016/j.scispo.2014.06.004

9. Jahic, D;; Begic, E. Exercise-associated muscle cramp - doubts about the cause. Mater Sociomed 2018, 30 (1), 67-9. doi:10.5455/msm.2018.30.67-69.

10. Maughan, R.J.; Shirreffs, S.M. Muscle cramping during exercise: causes, solutions, and questions remaining. Sports Med 2019, 49 (Suppl 2), 115-24. doi:10.1007/s40279-019-01162-1.

11. Swash, M.; Carvalho, M. Testing electrolyte supplementation for muscle cramp. Muscle Nerve 2019, 60 (5), $499-500$. doi:10.1002/mus.26686.

12. Schwellnus, M.P. Muscle cramping in the marathon: aetiology and risk factors. Sports Med 2007,37 (4-5), 364-7. doi:10.2165/00007256-200737040-00023.

13. Schwellnus, M.P.; Drew, N.; Collins, M. Increased running speed and previous cramps rather than dehydration or serum sodium changes predict exercise-associated muscle cramping: a prospective cohort study in 210 Ironman triathletes. Br J Sports Med 2011, 45 (8), 650-6. doi:10.1136/bjsm.2010.078535.

14. Nelson, N.L.; Churilla, J.R. A narrative review of exercise-associated muscle cramps: factors that contribute to neuromuscular fatigue and management implications. Muscle Nerve 2016, 54 (2), 177-85. doi:10.1002/mus.25176.

15. Giuriato, G.; Pedrinolla, A.; Schena, F.; Venturelli, M. Muscle cramps: a comparison of the two-leading hypothesis. J Electromyogr Kinesiol 2018, 41, 89-95. doi:10.1016/j.jelekin.2018.05.006.

16. Panza, G.; Stadler, J.; Murray, D.; Lerma, N.; Barrett, T.; Pettit-Mee, R.; Edwards, J.E. Acute passive static stretching and cramp threshold frequency. J Athl Training 2017, 52 (10), 918-24. doi:10.4085/1062-6050-52.7.03.

17. Miller, K.C.; Mack, G.W.; Knight, K.L.; Hopkins, J.T.; Draper, D.O.; Fields, P.J.; Hunter, I. Reflex inhibition of electrically induced muscle cramps in hypohydrated humans. Med Sci Sports Exerc 2010, 42 (5), 953-61. doi:10.1249/MSS.0b013e3181c0647e.

18. Simon, S.A.; de Araujo, I.E.; Gutierrez, R.; Nicolelis, M.A. The neural mechanisms of gustation: a distributed processing code. Nat Rev Neurosci 2006, 7 (11), 890-901. doi:10.1038/nrn2006.

19. Alvarez-Berdugo, D.; Rofes, L.; Farré, R.; Casamitjana, J.F.; Enrique, A.; Chamizo, J.; Padrón, A.; Navarro, X.; Clavé, P. Localization and expression of TRPV1 and TRPA1 in the human oropharynx and larynx. Neurogastroenterol Motil 2016, 28 (1), 91-100. doi:10.1111/nmo.12701.

20. Chang, R.B.; Waters, H.; Liman, E.R. A proton current drives action potentials in genetically identified sour taste cells. Proc Natl Acad Sci USA 2010, 107 (51), 22320-5. doi:10.1073/pnas.1013664107.

21. Huang, Y.A.; Maruyama, Y.; Stimac, R.; Roper, S.D. Presynaptic (Type III) cells in mouse taste buds sense sour (acid) taste. J Physiol 2008, 586 (12), 2903-12. doi:10.1113/jphysiol.2008.151233.

22. Wang, Y.Y.; Chang, R.B.; Allgood, S.D.; Silver, W.L.; Liman, E.R. A TRPA1-dependent mechanism for the pungent sensation of weak acids. J Gen Physiol 2011, 137 (6), 493-505. doi:10.1085/jgp.201110615.

23. de Ataide E Silva, T.; Di Cavalcanti Alves de Souza, M.E.; de Amorim, J.F.; Stathis, C.G.; Leandro. C.G.; Lima-Silva, A.E. Can carbohydrate mouth rinse improve performance during exercise? A systematic review. Nutrients 2014, 6 (1), 1-10. doi:10.3390/nu6010001.

24. Wickham, K.A.; Spriet, L.L. Administration of caffeine in alternate forms. Sports Med 2018,48 (Suppl 1), $79-91$. doi:10.1007/s40279-017-0848-2.

25. Fares, E.J.; Kayser, B. Carbohydrate mouth rinse effects on exercise capacity in pre- and postprandial States. J Nutr Metab 2011, 2011 (385962), 1-6. doi:10.1155/2011/385962.

26. Sinclair, J.; Bottoms, L.; Flynn, C.; Bradley, E.; Alexander, G.; McCullagh, S.; Finn, T.; Hurst, H.T. The effect of different durations of carbohydrate mouth rinse on cycling performance. Eur J Sport Sci 2014, 14 (3), 259-64. doi:10.1080/17461391.2013.785599.

27. Rennie, S. Electrophysical agents contraindications and precautions: an evidence-based approach to clinical decision making in physical therapy foreword. Physiother Can 2010, 62 (5), 1-3. doi:10.3138/ptc.62.5.

28. Miller, K.C.; Knight, K.L.; Wilding, S.R.; Stone, M.B. Duration of electrically induced muscle cramp increased by increasing stimulation frequency. J Sport Rehabil 2012, 21 (2), 182-5. doi:10.1123/jsr.21.2.182.

29. Stone, M.B.; Edwards, J.E.; Babington, J.P.; Ingersoll, C.D.; Palmieri, R.M. Reliability of an electrical method to induce muscle cramp. Muscle Nerve. 2003, 27 (1), 122-3. doi:10.1002/mus.10296.

30. Behringer, M.; Nowak, S.; Leyendecker, J.; Mester, J. Effects of TRPV1 and TRPA1 activators on the cramp threshold frequency: a randomized, double-blind placebo-controlled trial. Eur J Appl Physiol 2017, 117 (8), 1641-7. doi:10.1007/s00421-017-3653-6. 
31. Tanner, R.K.; Gore, C.J. Physiological tests for elite athletes, 2nd ed.; Australian Institute of Sport: Champaign, IL: Human Kinetics; 2013.

32. Ohno, M.; Lavender, A.P.; Sawai, A. Heat-induced body fluid loss causes muscle cramp during maximal voluntary contraction for the knee flexors. International Journal of Sport and Health Science 2018, 16, 191-9. doi:10.5432/ijshs.201729.

33. Hooper Marosek, S.E.; Antharam, V.; Dowlatshahi, K. Quantitative analysis of the acetic acid content in substances used by athletes for the possible prevention and alleviation of exercise-associated muscle cramps. J Strength Cond Res 2020, 34 (6), $1539-46$. doi:10.1519/JSC.0000000000003595.

34. Manning, C.; McDonald, P.; Murnaghan, C.; Bawa, P. Reciprocal inhibition versus unloading response during stretch reflex in humans. Exp Brain Res 2013, 226 (1), 33-43. doi:10.1007/s00221-013-3408-5.

35. Craighead, D.H.; Shank, S.; Alexander, L.M.; Kenney, W.L. Orally ingested transient receptor potential (TRP) channel activators attenuate the intensity-duration of voluntarily induced muscle cramps in humans. Faseb J 2016 30 (s1). doi:10.1096/fasebj.30.1_supplement.lb706.

36. Earp, J.E.; Stearns, R.L.; Stranieri, A.; Agostinucci, J.; Lepley, A.S.; Matson, T.; Ward-Ritacco, C.L. Electrolyte beverage consumption alters electrically induced cramping threshold. Muscle Nerve 2019, 60 (5), 598-603. doi:10.1002/mus.26650.

37. Craighead, D.H.; Shank, S.W.; Gottschall, J.S.; Passe, D.H.; Murray, B.; Alexander, L.M.; Kenney, W.L. Ingestion of transient receptor potential channel agonists attenuates exercise-induced muscle cramps. Muscle Nerve 2017, 56 (3), $379-85$. doi:10.1002/mus.25611.

38. Miller, C.K. Myths and misconceptions about exercise-associated muscle cramping. ACSMs Health Fit J 2016, 20 (2), 37-9. doi:10.1249/FIT.0000000000000187. 